\title{
Multi-robot Communication for Education and Research
}

\section{Mr. Robert L. Avanzato, Pennsylvania State University, Abington}

Bob Avanzato is an associate professor of engineering at the Penn State's Abington campus where he teaches courses in electrical and computer engineering, computer science, and information sciences and technology. His research interests are mobile robotics, intelligent systems, virtual world technology and innovative education. 


\title{
Multi-robot Communication for Education and Research
}

\begin{abstract}
Multi-robot communication is a key technology in modern robotics education and research. Applications such as search and rescue, exploration, mapping, reconnaissance, and remote data collection may all benefit from robot teams or swarms which rely on multi-robot communication technology. Based on the importance of this technology, our objective is to develop and evaluate educational resources to introduce multi-robot communication into the undergraduate experience using low-cost hardware and software tools. Using XBee radio technology (Digi International), educational resources, design challenges, and laboratories have been developed to support multirobot education for undergraduate engineering and information science students. A multi-robot design challenge for a regional robot contest, multi-robot classroom and laboratory activities, and a programmable controller for multi-robot communication are presented. The capabilities of these development tools also support multi-robot solutions across several low-cost mobile robot platforms, including VEX, Lego NXT, Arduino, and beyond. Based on the successes described, educators will have the tools to integrate multi-robot technology into the undergraduate STEM curriculum and outreach activities
\end{abstract}

\section{Introduction}

Multi-robot communication is a key technology in modern robotics education and research. There are many challenging and complex real-world problems which are best solved by teams of robots which are able to communicate, share resources and coordinate tasks. Applications, such as search and rescue, exploration, mapping, reconnaissance, and remote data collection may all benefit from robot teams or swarms, which rely on multi-robot communication technology. For example, researchers have proposed a robot swarm solution to effectively contain oil spills in the ocean. ${ }^{1}$ The robots, equipped with skimmers, use GPS and radio communication technology to coordinate operations. Once the spill is contained by the robot team, a ship can pump the oil into containers for removal and storage. In another research effort, the coordination of a ground robot and a micro aerial robot is leveraged in search and rescue operations in an unknown environment. ${ }^{2}$ The aerial robot gathers visual data which can be communicated to the ground robot vehicles to enable the ground vehicles to more quickly and efficiently navigate and locate targets and potentially locate victims. Luca, et al provide a taxonomy and overview of multirobot trends and applications in the space and defense application areas. ${ }^{3}$ Advantages of multi- 
robot solutions in application areas such as mine clearance, planetary resource scavenging, emergency response and convoy transport are included. These researchers also mention the growing importance of the human operator in multi-robot systems, and that much technology needs to be developed in the area of multi-robotic systems in general.

Due to the importance of multi-robot solutions, it is equally important that undergraduate education and undergraduate research facilities have access to low-cost, reliable and robust robot communication technology, and have access to the associated educational resources in order to effectively expose this technology to students. As an example of existing educational initiatives, educators at Rice University have recently developed and deployed low-cost robots that support problem-based learning and foster student collaboration with multi-robot assignments and projects. ${ }^{4}$ The multi-robot approach has been successfully incorporated into a freshman introduction to engineering course, an advanced robotics course, and outreach activities. The group designed a small, low-cost robot, programmed in Python, which has ample sensors and communication capabilities. The customized robot platform is inexpensive enough to support a “one-robot-per-student" implementation. Applications such as Monte Carlo localization and particle filters have been realized with this robotics system.

Computer science researchers at Carnegie Mellon University (CMU) and CMU's Robotics Academy actively support multi-robot technology education through tutorials, design challenges, programming examples, instructional videos, and teachers' resources. ${ }^{5}$ One of the goals is to promote interest in computer science and STEM careers among a K-12 audience. The multirobot resources and technology are designed with the ROBOTC programming tool and XBee (Digi International) radio technology. XBee technology is a low-cost, low power, radio technology that supports wireless serial communication, with a range up to 300 feet. ROBOTC is a powerful C-based robotics software development environment which currently supports Lego NXT, VEX, and Arduino robot platforms. ${ }^{6}$ Although primarily targeting K-12, the ROBOTC programming tool (which offers a real-time debugger, multitasking, etc.) is sophisticated enough for use in undergraduate engineering programs. Additionally, ROBOTC allows educators to use the same programming language environment to engage students in multi-robot communication among teams of similar robots and also across robot platforms. For example, it is relatively straightforward to use ROBOTC to support communication between an Arduino-based robot and an NXT-based robot. This author spent 8 weeks at the CMU Robotics Academy participating in the multi-robot initiative in the fall of 2011, and many of the multirobot educational projects described in this paper have been motivated by that experience. Of course, other robot programming languages, in addition to ROBOTC, support XBee wireless communication technology as well.

This paper will describe the application of XBee radio technology and a variety of software tools to develop educational resources, laboratory exercises and projects to implement multi-robot communication learning experiences for undergraduate engineering and information science students. These educational resources and case studies include: 1) a multi-robot design challenge 
for a regional robot contest, 2) multi-robot classroom activities for an introductory robotics module and a computer science course, and 3) a programmable wireless controller for multirobot communication. The capabilities of these development tools also support multi-robot solutions across several commonly-used mobile robot platforms, including VEX, Lego NXT, and Arduino systems. The overall objective is to provide educators with successful tools and resources to integrate multi-robot communication and technology into the curriculum and outreach activities.

\section{Multi-robot Educational Developments}

\subsection{Multi-robot Design Challenge for a Robot Contest}

Penn State Abington College has been offering a regional autonomous firefighting robot contest each spring since 1996. ${ }^{7}$ The contest is based on the Trinity College Home Firefighting robot contest $^{8}$ and it challenges a single autonomous robot to navigate a maze consisting of 4 rooms, locate a candle, and extinguish a single, randomly-located candle in a minimum of time. The contest is open to K-12, college, and beyond and generally attracts 40 robots or more annually at our regional campus event. This contest design challenge serves as an outreach effort and is also used as a course team project for several lower division engineering and computer science courses. In spring 2012, an optional multi-robot challenge was developed for use at our regional contest to promote interest in multi-robot system education and research. ${ }^{7}$

Here is a summary of the multi-robot challenge developed for our regional firefighting contest:

1. Robot team must consist of 2 or more robots. Robot communication and coordination must be demonstrated. (The maximum size of each robot is $12 \mathrm{in}$. by $12 \mathrm{in}$. by $12 \mathrm{in}$.)

2. Two lit candles (instead of one) will be located in 2 random rooms of the maze. The goal is to extinguish both candles in the minimum amount of time.

3. The starting area for the robot team will be expanded to accommodate multiple robots.

4. Only one robot in the team will be started manually -- the other robots must start based on communication with this single, manually-started robot.

5. All of the robots in the robot team must be "aware" when both candles have been extinguished. That is, each robot in the team must clearly indicate an "All Clear" message (either audible or displayed on a screen, or equivalent) when the goal is achieved.

A set of teams in an Information Sciences and Technology (IST) special topics course in robotics participated in this autonomous multi-robot challenge in the spring of 2012. Each of the seven teams in the class was tasked with designing a single robot to participate in the firefighting regional challenge. Three teams out of the seven elected to additionally participate in the multirobot challenge. There were a total of four robots in the multi-robot team, which included three VEX robots and a single Lego NXT robot. Each robot was equipped with an XBee radio module and all software (for all platforms) was developed using the ROBOTC software tool. Each VEX 
robot explored a portion of the maze. The NXT robot was stationary and provided master control and coordination of the other robots. Figure 1 below shows the four robots in the start area, and figure 2 shows the robot team as well as an example of how the two candles might be configured prior to a run.

The basic software algorithm for the NXT master robot is listed below:

1. Send message to each of the three VEX robots in sequence (ping). Wait for a response message from each robot. This step verifies active communication with all of the robots prior to start.

2. Send message to start each of the three VEX robots in a specified sequence.

3. Listen for messages from any of the VEX robots that a candle has been located and extinguished. If a candle is extinguished by a VEX robot, then increment a counter by 1 .

4. If the counter is equal to 2 , then both candles have been extinguished and the goal is complete. Send "All Clear" message to each VEX robot for display.

5. Display "All Clear" message on NXT robot and wave green flag.

Because all of the robots were on the same channel, any radio message sent by any one of the robots would be received by all of the other robots. In order to direct messages to a particular robot, a robot identifier was placed in each of the string messages. Each robot checked the robot name identifier to determine whether to accept or reject the message. For example, "VEX1 Move" would only command the robot uniquely named VEX1 to move, and "VEX2 Move" would similarly control the robot named VEX2, etc. The multi-robot team was able to demonstrate a successful completion of the multi-robot challenge. The significance of this achievement is that this junior-level IST robotics class consisted of nonengineering students with no prior background in robotics. Although the team did receive support from the instructor, the use of ROBOTC software for the VEX and NXT and XBee support was judged to be an important element in the overall success of the project. Another interesting outcome of this multi-robot effort was that it required collaboration among several of the student design teams (as well as collaboration within each team.) This dynamic team collaboration with multi-robot design has also been noted by the Rice University educators. ${ }^{4}$

The Firefighting contest is an ideal design problem to demonstrate the advantages and challenges of a multi-robot solution. The multi-robot technology was successfully demonstrated at a campus robot contest to a wide audience of students, teachers, and parents. Although only two senior entries participated in the 2012 multi-robot design option, we will continue to offer this challenge in future regional robotics contests, and we hope to encourage K-12 participation in the near future. 


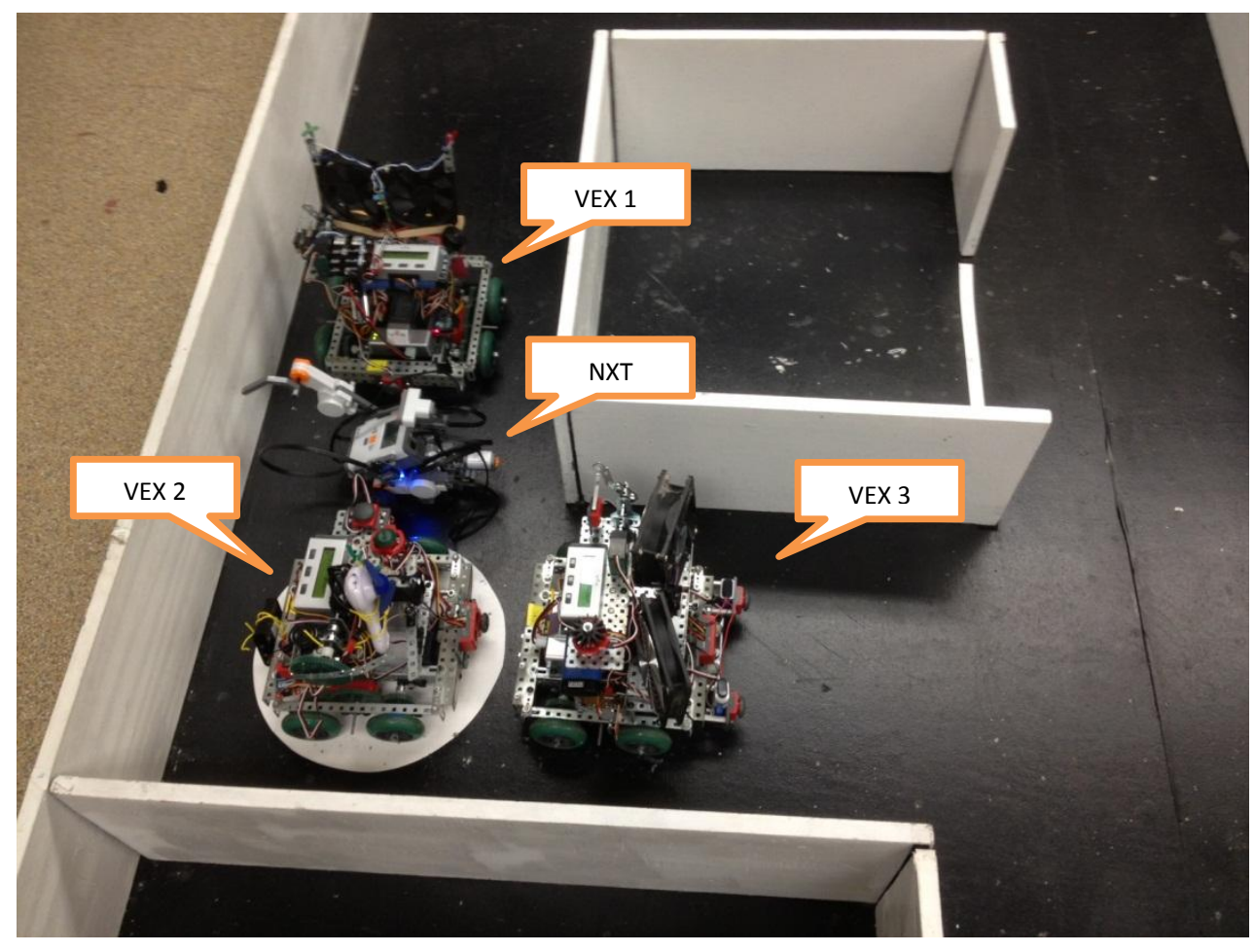

Figure 1. Multi-robot Team in Start Area

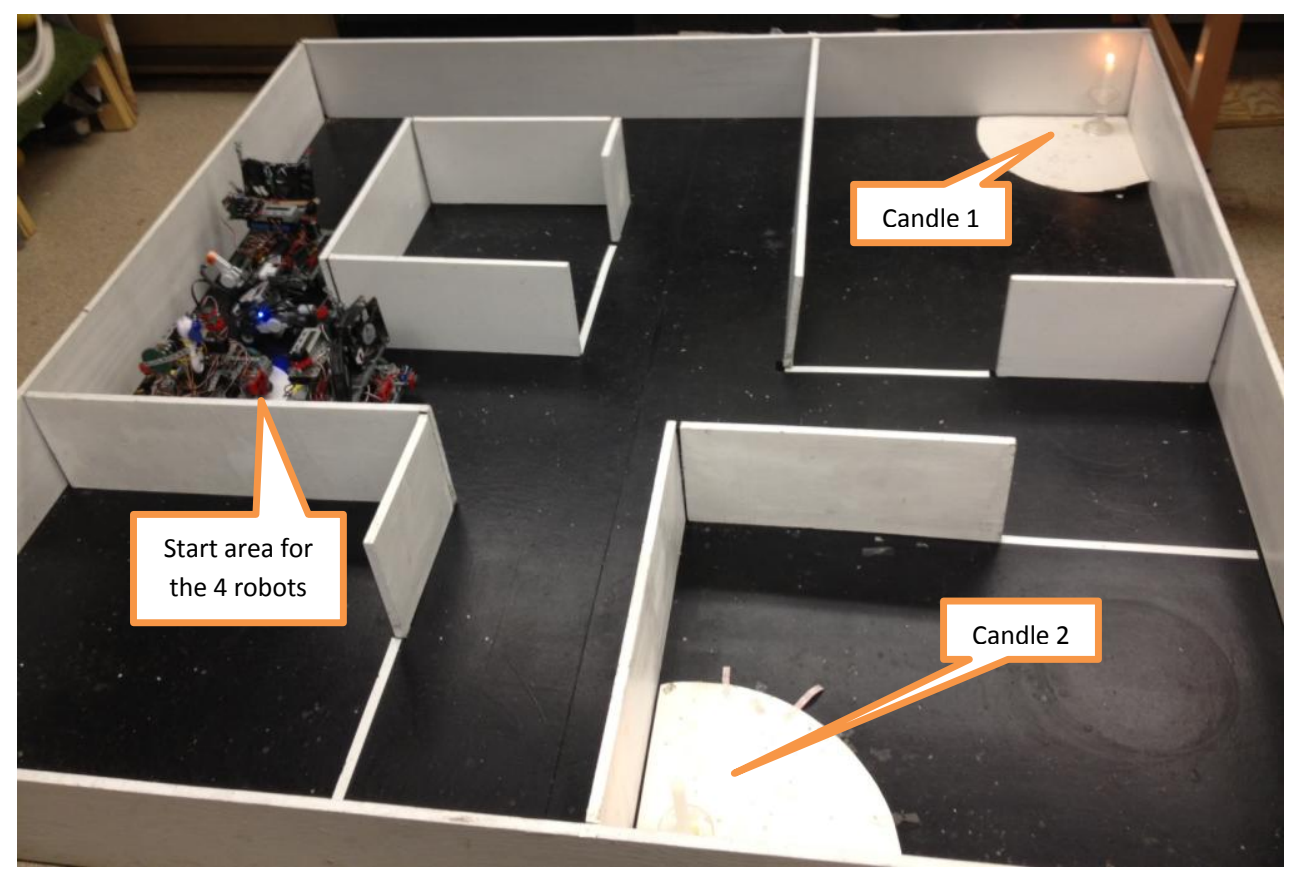

Figure 2. Multi-robot Team in Maze with Candles

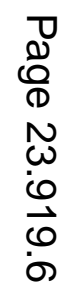


There is also existing sample code for detection of robot identification codes within a message, and the encoding and decoding of numeric data, such as sensor data, that can then be shared wirelessly among a team of robots. Based on these basic exercises, two of the student teams designed more sophisticated working prototypes of multi-robot systems. One example is a pair of dancing robots with motion synchronized and sequenced with multi-robot communication and another example is a sweeper robot and dustpan robot (figure 5). Overall, the multi-robot exercises were very successful with the freshman class and the multi-robot projects developed speak to the simplicity of establishing radio communication between two robots using the software and hardware tools described.

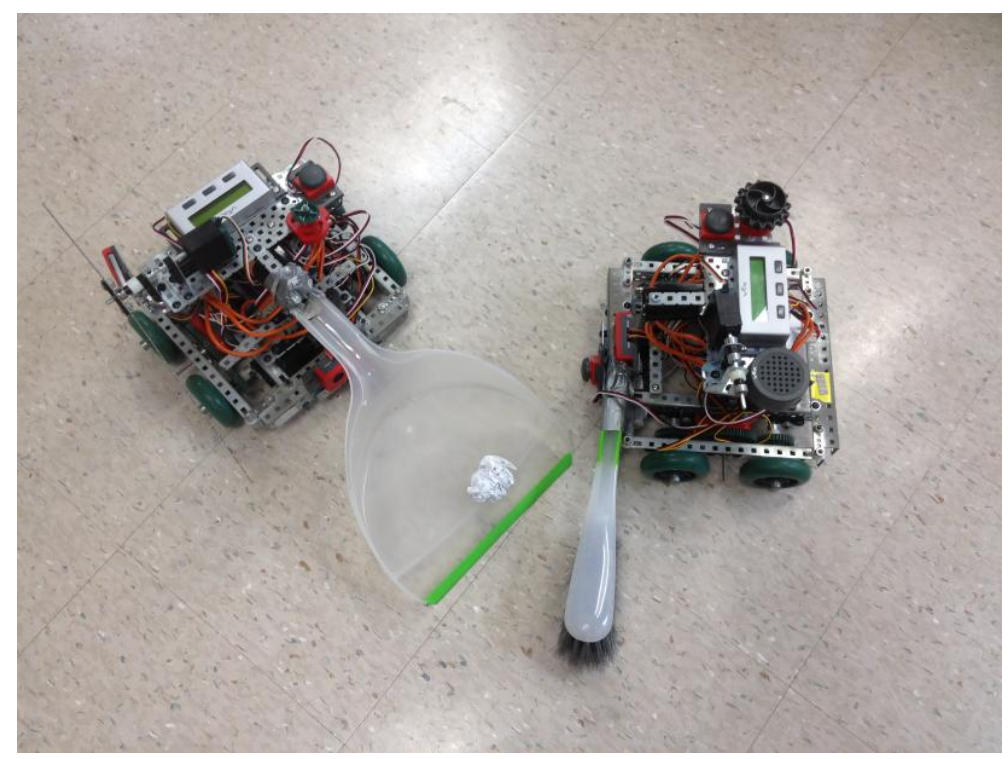

Figure 5. Floor sweeping multi-robot prototype

Students in an introductory computer science course for engineering majors were also exposed to the multi-robot technology in the spring of 2013 using similar hardware and software tools. Multi-robot lab experiences were successfully completed by the student teams within 3 to 6 hours of laboratory time. Examples of multi-robot team projects developed in this course included sharing of sensor data to navigate a team of two robots, and using sensors on one robot (such as a potentiometer) to remotely control the motion (steering) of a second robot using XBee radio communication. In addition to the high success rate for project completion, the levels of student enthusiasm and student team interactions were also observed to be very high during the multi-robot laboratory experiences.

\subsection{Programmable Multi-robot Controller}

In the third educational project to be presented, a low-cost, handheld, programmable remote control device (using XBee) has been designed and constructed to allow a human operator to wirelessly control the motion of one robot or multiple robots in a team, and also allow the collection and display of sensor data sent back from any of the robots. The same XBee technology and programming tools used in the robots is also used for the controller. In this case 
we have used an Arduino UNO as the microcontroller for the handheld controller, and we have programmed the controller device with the Arduino software. ${ }^{9}$ The Arduino is an open source microcontroller solution which features low-cost hardware, free software development tools, and a large community of users. The goal of this project is to allow students to program and customize the controller to adapt it to a wide range of creative multi-robot (and cross-platform) applications in which a human operator is a key factor. The controller was developed with enough functionality to allow a lower-division (and beyond) engineering students to expand the operation of the unit. The controller is shown in figure 6 , and in its present configuration has 4 pushbuttons to control the motion of remote robots (forward and reverse, right and left motors), and a LCD screen with a potentiometer to advance and select the menu options to be displayed on the screen. An XBee device is mounted on the external enclosure of the controller device. This particular controller was tested with an Arduino-based robot (also equipped with an XBee device) as shown in figure 7. The Arduino was also programmed using the Arduino software tools, but it should be mentioned that with a few hardware changes, both the controller and the Arduino robot could be fully programmed using ROBOTC. This controller can be used to communicate with any other technology (such as a NXT or VEX robot) that uses XBee technology. The menu system programmed into the initial, tested prototype controller is as follows:

- $\quad$ Ping Arduino

- $\quad$ Ping VEX

- Ping NXT

- $\quad$ Ping All

- Move Arduino

- Move VEX

- Move NXT

- Move All

- Sonar Arduino

- Sonar VEX

- Sonar NXT 


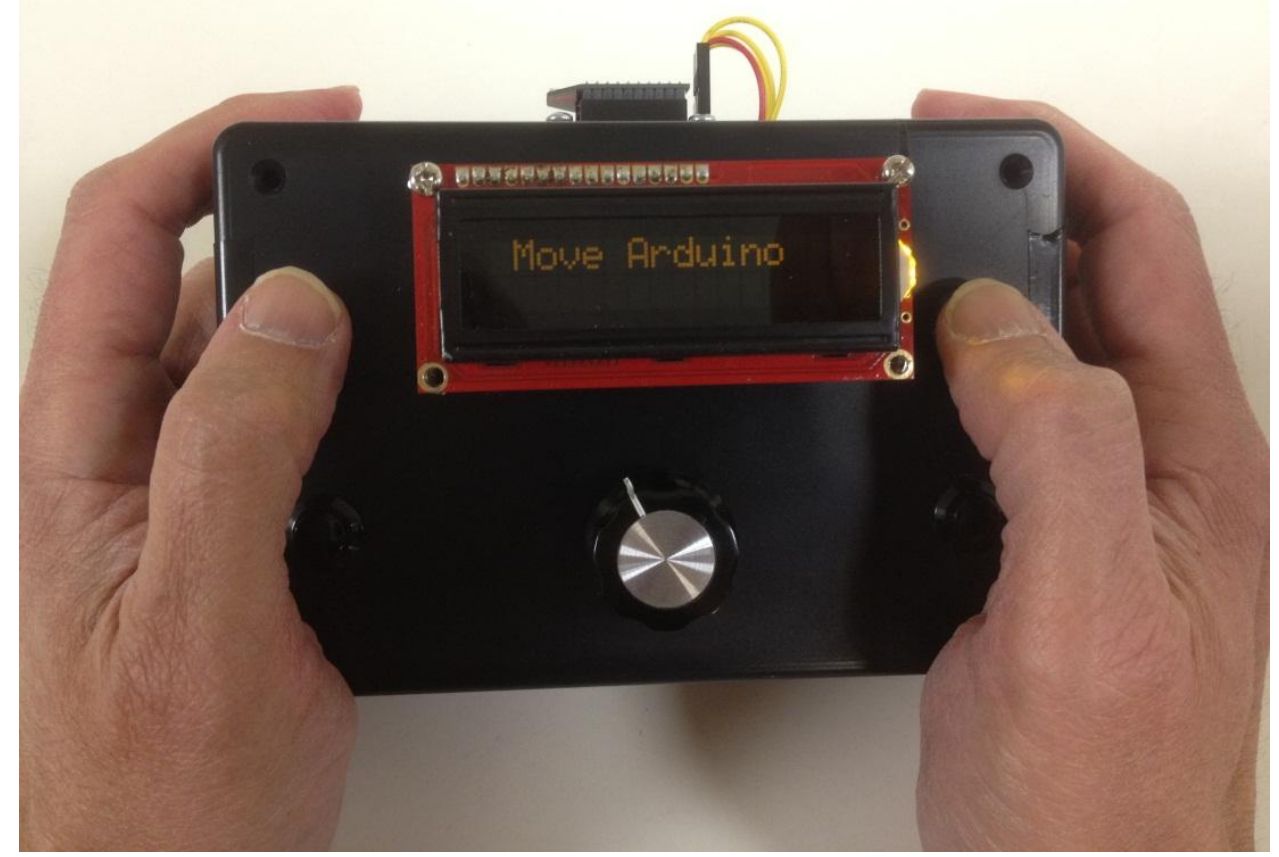

Figure 6. Arduino-based XBee Controller

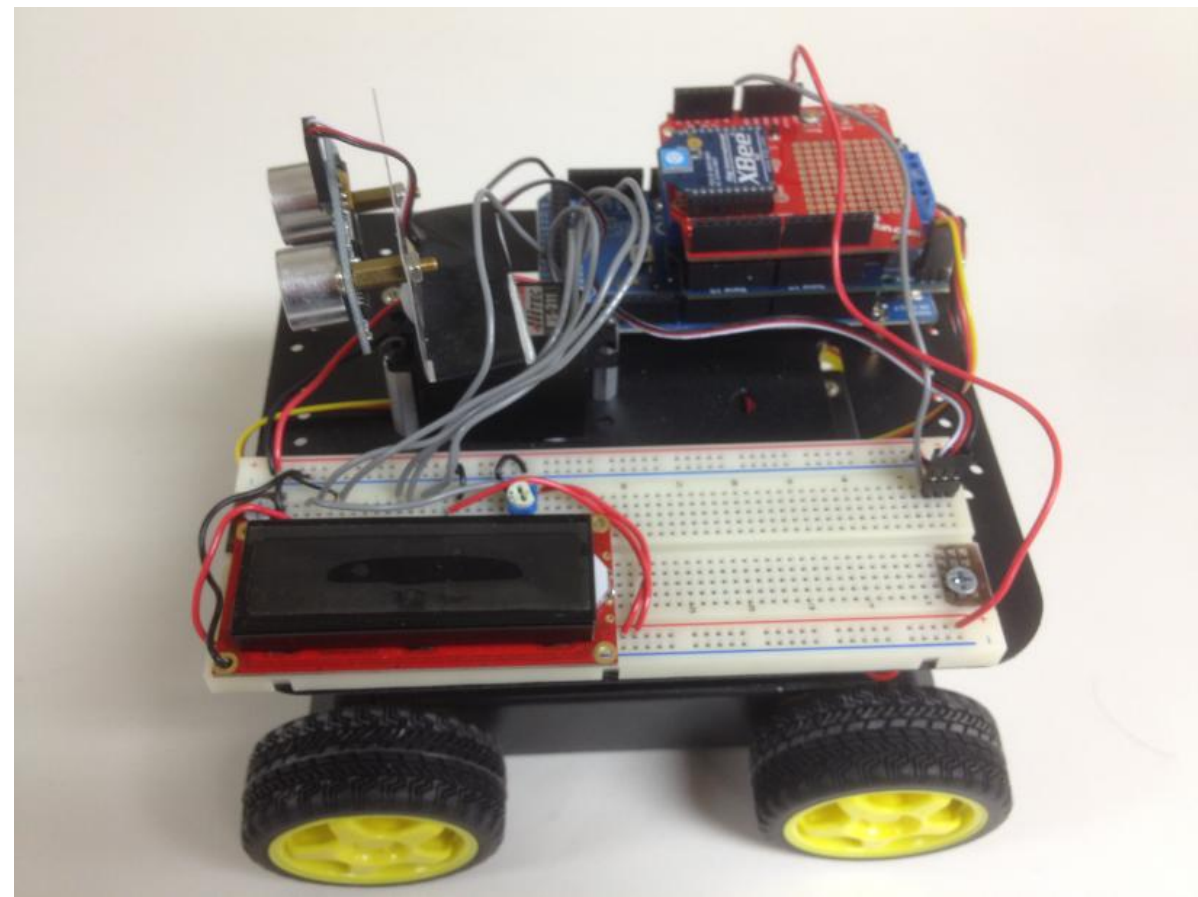

Figure 7. Arduino-based mobile robot with XBee

The sonar command should result in sonar sensor readings from a selected robot to be displayed on the handheld controller LCD. The controller menu, display and button responses can be redesigned and reprogrammed to meet many educational and project objectives. We are 
planning to use this controller and software as a platform for student-based projects with undergraduate engineering students in the near future.

\section{Conclusions}

Multi-robot communication and coordination is an important area for the future of robotics and robotics education. The availability of low-cost, wireless technology and high-level software support for existing educational robot platforms brings multi-robot applications and research within the reach of many educators and students. There are several key findings based on the results presented in this paper: 1) multi-robot technology is accessible to a wide range of students including engineering and non-engineering majors, and K-12; 2) the multi-robot technology presented supports existing, low-cost educational robot platforms and also cross-platform designs; 3 ) the multi-robot technology can be successfully introduced into outreach activities such as the robot contest challenge described above; and 4) the multi-robot projects encourage a dynamic collaboration among student design teams. We look forward to developing more advanced multi-robot curriculum and projects to build on these results. It is hoped that the presentation and discussion of these successful education applications, resources and case studies of multi-robot communication systems will provide a useful starting point for educators in engineering and related fields.

\section{References}

[1] Zahugi, E., et al, "Design of Multi-Robot System for Cleaning Up Marine Oil Spill," International Journal of Advanced Information Technology, Vol. 2 (4), p. 33-43, 2012.

[2] Luo, C., et al, "Multi-robot Search and Rescue Team," Proceedings of the 2011 IEEE International Symposium on Safety, Security and Rescue Robotics Kyoto, Japan, November 1-5, p 296-301, 2011.

[3] Lucas, N., "Review of Multi-robot Taxonomy, Trends and Applications for Defense and Space," Proc. of SPIE, Vol. 838783871 N-1, 2012.

[4] McLurkin, James, et al, "Using Multi-Robot Systems for Engineering Education: Teaching and Outreach With Large Numbers of an Advanced, Low-Cost Robot," IEEE Transactions on Education, preprint, [accessed 12-302012], 2012.

[5] CMU Multi-Robot CS2N website. http://www.cs2n.org/activities/multi-robot-communications (accessed 1-42013)

[6] ROBOTC website. http://www.robotc.net/ (accessed 1-4-2013).

[7] Penn State Abington Regional Fire Fighting Robot Contest website: http://www.cede.psu.edu/users/avanzato/robots/contests/firefighting/index.htm (accessed 1-4-2013). 
[8] Trinity College Fire Fighting Home Robot Contest website. http://www.trincoll.edu/events/robot/ (accessed 1-42013).

[9] Arduino project website: http://www.arduino.cc/ (accessed 1-4-2013). 